Existence theorem of the quadruple ( $P, R, F, M)$ : Precision, Recall, Fallout and Miss

Peer-reviewed author version

EGGHE, Leo (2007) Existence theorem of the quadruple (P, R, F, M): Precision, Recall, Fallout and Miss. In: INFORMATION PROCESSING \& MANAGEMENT, 43(1). p. 265-272.

DOI: $10.1016 / j . j p m .2006 .03 .026$

Handle: http://hdl.handle.net/1942/754 


\section{Existence theorem of the}

\section{quadruple (P, R, F, M):}

\section{Precision, Recall, Fallout and Miss}

by

L. Egghe

Universiteit Hasselt (UHasselt), Campus Diepenbeek, Agoralaan, B-3590 Diepenbeek,

Belgium $^{1}$

and

Universiteit Antwerpen (UA), Campus Drie Eiken, Universiteitsplein 1, B-2610 Wilrijk,

Belgium

leo.egghe@uhasselt.be

${ }^{1}$ Permanent address

$\underline{\text { Key words and phrases: }}$ precision, recall, fallout, miss, universal IR surface, existence 